\title{
Challenges in medical education: a social network approach to health
}

\author{
Gary L. Albrecht ${ }^{1}$
}

As public health, medicine and health-care systems grow in a global, inter-connected context, medical educators are challenged to produce conceptual frameworks that organize the vast knowledge accumulated (1). What has become apparent is that no single, traditional discipline or viewpoint holds the key to understanding health in today's world $(2,3)$. Consequently, considerable attention is being given to integrating the multiple and diverse contributions of health science, prevention and practice into a single framework (4-6). The concept of social networks provides an organizational model for integrating the contributions of biomedical, social and computational sciences into a single paradigm that can help us to better understand health dynamics, practices and delivery.

Social networks portray the set of inter-relationships between humans as they interact with each other in their daily personal, work and public lives. Social networks provide the social glue of human experience. They map the connections that individuals have with one another and also indicate how groups of people relate to each other. They describe the social structure formed by a complex set of social connections that exist in family, friendship and work groups, and they can be extended to study health and help-seeking networks, medical practices, diffusion of health practices and spread of disease and infections. Social network theory, techniques, analysis and applications are well explicated in the work of Christakis and Fowler (7). Using diverse data sets, the authors show how obesity (and the resulting risk of diabetes) is spread through social networks, smoking cessation is influenced by network ties, social network sensors can be used in the early detection of contagious disease outbreaks and social network connections can predict heavy drinking, loneliness, depression, happiness, eating behaviours, levels of physical activity and sleep patterns $(8-10)$. This work has been extended to understand the spread of HIV/AIDS, utilization of vaccines, engendering healthy behaviours and diffusing medical innovations and health interventions (11). In a sense, people behave like those around them and so interventions ought to be designed to influence the social network not just the individual.

The social network approach to health and medicine is important because it conceptually organizes much of what we know about health practices, suggests effective public health programmes and policies and reorients how we train health-care workers. The approach is useful because it equally applies to prevention, monitoring and dealing with infectious and chronic diseases. This conceptual framework also is applicable to understanding how biological processes are influenced through inheritance and environmental exposure and how cyberconnections via Facebook, Twitter, chat groups, professional newsletters and blogs affect people's knowledge, behaviour and normative actions.

Applying a social network approach to medicine and health care requires changes in medical education and practice. The focus moves from concentrating on individual patients and their outcomes to social constellations of people related by social ties. To utilize these insights, physicians and health-care workers need to be trained to identify social networks important to the individual and the community. These social networks radiate outwards from the original individuals contacted. Indeed, the patient seen may have a significant influence on the attitudes, values, behaviour and health of others who are up to three steps removed in a network. There are influential persons in these networks who serve as nodes in the network and have a disproportionate influence on others in the constellation. To take advantage of these network dynamics, physicians and other health-care workers need training in the classroom and community to learn how to identify salient networks in the family and community, find the influential persons in the node positions and determine how best to nudge these individuals into healthier behaviours which they, in turn, will transmit throughout their networks. More attention to social science advances promoting behavioural change and a deeper understanding of epidemiology would provide the tools for social network interventions. Substantial supervised field work on specific community problems would be a key component of this revised medical education. Examples of these efforts might be to ensure that all babies are born in hospitals or clinics, 
vaccinations are delivered throughout the community to those most at risk, epidemics are monitored and halted early in their tracks, and smoking cessation, healthier eating and cooking habits and increased daily exercise are promoted through the social networks in the community. To facilitate these interventions, more women would likely need to be trained as physicians and health assistants, as they would be more readily accepted within women's social networks to work on woman, maternal and child health issues. HIV-positive persons can be trained as health-care workers to access HIV/AIDS social networks in order to encourage testing, medical regimens and practices such as safe sex and clean needle use to control the spread of the disease. Similar efforts can be used to increase environmental awareness of conditions that increase the risk of asthma, cholera, tuberculosis and trauma due to unsafe work conditions and road accidents. In sum, a social network approach to health and medicine targets conditions embedded in society and alters the focus of health-care workers from individuals to social networks that exist in the community. This places a premium on prevention, education and monitoring conditions in the community, changes the unit of analysis from the individual to the community, and moves much health-care work out of hospitals and clinics into neighbourhoods. The next challenge of medical education concerns how to effectively make use of the power of social network approaches to health care using modern biomedical and information technology.

\section{References}

1. Fineberg HV. Public health and medicine where: the twain shall meet. Am J Prev Med. 2011 Oct;41(4) Suppl 3:S149-51. PMID:21961655

2. Mabry PL, Olster DH, Morgan GD, Abrams DB. Interdisciplinary and systems science to improve population health: a view from the NIH Office of Behavioral and Social Sciences Research. Am J Prev Med. 2008 Aug;35(2 Suppl):S211-24.

3. Thibault GE. Reforming health professions education will require culture change and closer ties between classroom and practice. Health Aff (Millwood). 2013 Nov;32(11):1928-32. PMID:24191082

4. Farmer PE. Shattuck Lecture. Chronic infectious disease and the future of health care delivery. N Engl J Med. 2013 Dec 19;369(25):2424-36. PMID:24350951

5. Langstrup H. Chronic care infrastructures and the home. Sociol Health IIIn. 2013 Sep;35(7):1008-22. PMID:23301691

6. LaPelle NR, Dahlen K, Gabella BA, Juhl AL, Martin E. Overcoming inertia: increasing public health departments' access to evidence-based information and promoting usage to inform practice. Am J Public Health. 2014 Jan;104(1):77-80. PMID:24228662

7. Christakis NA, Fowler JH. Connected: the surprising power of our social networks. New York: Little, Brown and Co.; 2009.

8. Christakis NA, Fowler JH. The spread of obesity in a large social network over 32 years. N Engl J Med. 2007 Jul 26;357(4):370-9. PMID:17652652

9. Christakis NA, Fowler JH. The collective dynamics of smoking in a large social network. N Engl J Med. 2008 May 22;358(21):2249-58. PMID:18499567

10. Christakis, NA, Fowler, JH. Social network sensors for early detection of contagious outbreaks. PLoS One. 2010 Sep 15;5(9):e12948.

11. Christakis NA, Fowler JH. Social contagion theory: examining dynamic social networks and human behavior. Stat Med. 2013 Feb 20;32(4):556-77. PMID:22711416 